\title{
Effects of JPEG and JPEG2000 Compression on Face Recognition
}

\author{
Kresimir Delac, Mislav Grgic, and Sonja Grgic \\ University of Zagreb, FER, Unska 3/XII, Zagreb, Croatia \\ kdelac@ieee.org
}

\begin{abstract}
In this paper we analyse the effects that JPEG and JPEG2000 compression have on subspace appearance-based face recognition algorithms. This is the first comprehensive study of standard JPEG2000 compression effects on face recognition, as well as an extension of existing experiments for JPEG compression. A wide range of bitrates (compression ratios) was used on probe images and results are reported for 12 different subspace face recognition algorithms. Effects of image compression on recognition performance are of interest in applications where image storage space and image transmission time are of critical importance. It will be shown that not only that compression does not deteriorate performance but it, in some cases, even improves it slightly. Some unexpected effects will be presented (like the ability of JPEG2000 to capture the information essential for recognizing changes caused by images taken later in time) and lines of further research suggested.
\end{abstract}

\section{Introduction}

With the growing number of face recognition applications in everyday life, image- and video-based recognition methods are becoming more and more important research topic [1]. Effects of pose, illumination and expression are issues most studied in face recognition so far. Very little has been done to investigate the effects of compression on face recognition. Still-to-still image experimental setups are often researched but only in uncompressed image formats. Still-to-video research mostly deals with issues of tracking and recognizing faces in a sense that still uncompressed images are used as a gallery and compressed video segments are probes. Effects of compression are rarely discussed in such papers and rarely researched in general because there is a general belief that the effect of compression in machine vision applications is deleterious. The compression is, therefore, often avoided. Since surveillance cameras and other image acquisition equipment often give their output in a compressed format, exploring compression effects on known face recognition algorithms seems like a reasonable line of research and that is the one we will pursue in this paper. Another important issue would be ability to store compressed face images (without performance degradation when subject to recognition) on a low-capacity chips and smart cards. This would be a great advantage and would contribute to faster implementation of biometrics in every day life (a good example is the e-passport). 
In this paper we will compare different face recognition algorithms' behaviour in a still-to-still setup with uncompressed training and gallery images and probe images compressed with various compression ratios. This setup mimics the expected real-life circumstances where the image captured by a surveillance camera is probed to existing high-quality gallery images. Algorithms tested in this paper are wellestablished subspace face recognition projection methods: Principal Component Analysis (PCA) [2], Independent Component Analysis (ICA) [3] and Linear Discriminant Analysis (LDA) [4], combined with common distance metrics (L1, L2 and cosine) in a nearest-neighbour matching system. Bits per pixel (bpp) will be a measure of compression for both tested compression algorithms: JPEG [5] and JPEG2000 [6]. It will be shown that compression does not significantly affect performance even at 0.2 bpp (a 40:1 compression). Actually, in many cases the performance goes slightly up for some compression ratios.

The rest of this paper is organized as follows: Section 2 gives an overview of previous work, Section 3 describes experimental setup used in our research, Section 4 reports results and analyses them and Section 5 concludes the paper.

\section{Previous Work}

FRVT 2000 [7] tried to estimate the effects of lossy image compression on the performance of face recognition algorithms by minimising a situation in which the gallery images were obtained under favourable, uncompressed circumstances, but the probe sets were obtained in a less favourable environment in which compression was applied. They used JPEG compression and tested algorithms with dupl probe set with images in it compressed to $0.8,0.4,0.25$ and $0.2 \mathrm{bpp}$. With this setup, they concluded that compression does not adversely affect performance and that the performance of algorithms drops significantly only with images compressed below $0.2 \mathrm{bpp}$. In their experiment the recognition rate goes up slightly for compression ratios of 10:1 (0.8 bpp) and 20:1 (0.4 bpp). In conclusion, they recommend that additional studies on the effect of compression be conducted as their results are aggregated and only consider JPEG compression. This paper was the main motivation for our research.

Wat \& Srinivasan [8] explored the effects of JPEG compression on PCA and LDA with the same setup as in FRVT 2000 (compressed probes, uncompressed gallery). Results were presented as a function of JPEG quality factor and are therefore very hard to interpret (the same quality factor will result in a different compression ratios for different images, dependent on the given image's statistical properties). By using two different histogram equalization techniques they claim that there is a slight increase in performance with the increase in compression ratio for LDA in the illumination task ( $f c$ probe set). For all other combinations the results remain the same or decrease with higher compressions. This is in slight contradiction with results obtained in FRVT 2000.

Moon \& Phillips [9] examined the effects of both JPEG and wavelet compression (no details on wavelet compression were given). The original images were compressed and then uncompressed prior to being processed by the normalization step. For both compression methods, the images were compressed to $0.5 \mathrm{bpp}$. The 
standard PCA+L1 algorithm was tested with eigenvectors derived using uncompressed images. Results show no degradation of performance for JPEG and a slight (questionably significant) increase in performance for wavelet compression (for $d u p l$ and $f b$ sets).

As can be seen, none of the above papers used standard JPEG2000 compression and none gives a comprehensive study across various probe sets and for a larger number of algorithms. By experimenting with standard JPEG and JPEG2000 compression techniques over a wide range of compression ratios, we will give the first comprehensive comparison of the influence of those two techniques on recognition performance, across 12 different subspace face recognition algorithms.

\section{Experimental Setup}

Our experiment was performed on a standard grey FERET data set [10], consisting of images of 1196 individuals taken under various conditions and at various points in time. Also, to achieve highly reproducible results, standard test sets were used, i.e. $f b$ (different expression test), $f c$ (different illumination), dupl (images taken anywhere between one minute and 1,031 days after the gallery image) and dup2 (images taken at least 18 months after the gallery image was taken). By using all four sets, our results will present a substantial expansion of FRVT 2000 compression experiment.

All images in these subsets were compressed using JPEG and JPEG2000 compression techniques, with various compression ratios (bitrate, bpp): $0.1,0.2, \ldots$, 1.0 bpp. To compress images using JPEG, the Independent JPEG Group's JPEG software packet (JPEG6b32) [11] was used. To yield various bitrates, quality parameter was iteratively set until the desired bitrate was achieved. Due to the relative simplicity of face images in FERET database, it was impossible to compress some of the images to exactly $0.1 \mathrm{bpp}$. In those cases, the lowest possible bitrate was used. The bitrates thus varied from 0.1 to about 0.15 in some cases. For the sake of clarity we will refer to all those bitrates as $0.1 \mathrm{bpp}$ in further text. To compress images using JPEG2000 standard, a Kakadu V4.2 (up to date with Part 1 of the JPEG2000 standard) [12] was used with the switch "-rate" set to a required bitrate value. For JPEG2000 there was no trouble achieving the exact predefined bitrates.

Compression was done on original images of size of $256 \times 384$ pixels. After compression, all images (compressed and uncompressed) were rotated (using affine transformations with bilinear interpolation) to align the eyes at a fixed location across all images, cropped to the size of $128 \times 128$ pixels and histogram equalized to values 0 to 255 (see Figure 1). It is important to mention that all compressed images were uncompressed prior to recognition stage, thus, the recognition was done in pixel domain.

Algorithms were trained using uncompressed images of 225 individuals for which there were exactly 3 images per person in the data set. Thus, the training set consists of 675 images. This set of images overlaps with the query sets in the following manner: 224 images are in the gallery ( $f a$ set), another 224 images are in the $f b$ set and of the same subject as the ones taken from the gallery. Further 3 images are from the dupl set and the rest 224 images are not in any set used in the recognition stage. After 
training, a 270-dimensional subspace (224-dimensional for LDA) was derived retaining more than $95 \%$ of the original information. Recognition was done in those subspaces by standard nearest neighbour matching with L1, L2 and cosine (COS) metrics.
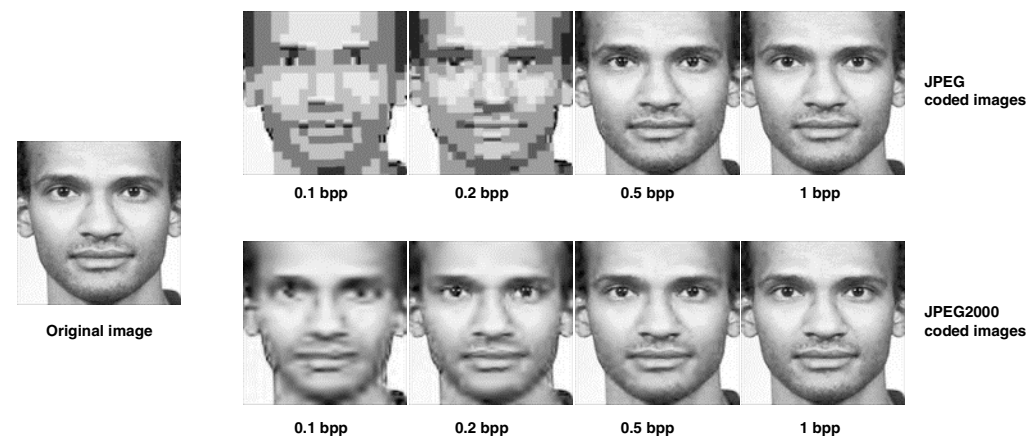

Fig. 1. Example of image degradation when subject to compression

\section{Results and Analysis}

We tested all 12 algorithms with compressed probe images in four standard test sets against uncompressed gallery images. As can be seen, results obtained using compressed probe images (Tables 1-4) are comparable to the ones obtained using uncompressed probe images (the rightmost column in all tables). The values in tables present rank 1 recognition percentage. The conclusion of FRVT 2000 that performance goes slightly up for compression ratios of 10:1 (0.8 bpp) and 20:1 (0.4 bpp) is confirmed in our experiment. Actually, we also show that in many other cases performance goes slightly up for compressions between 0.2 and $0.8 \mathrm{bpp}$. These cases are bolded in all tables. Our results are in some disagreement with Wat \& Srinivasan because we found that the performance goes up with compression in quite a few cases and not just for LDA. Moon \& Phillips' results are confirmed here also. All this gives us the reason to believe that our conducted experiments are consistent with previous studies so we can give a relevant contribution for a wide range of bitrates. Some recognition performance results obtained by other authors with JPEG compression will be confirmed and expanded. In addition, new results using JPEG2000 will be reported, making this paper a first comprehensive study of the effect of JPEG2000 compression on face recognition.

If you take a closer look at the results shown in tables, you will observe that in 36 out of possible 48 cases, the performance goes up for one or more compression ratios and compression techniques tested. Even though the difference is often not statistically significant (we proved this by using McNemar's hypothesis test - details given in Appendix) we believe that this is an important result as it encourages further research into the theoretical properties of both compression and recognition algorithms that led to this performance improvement. 
Table 1. Rank 1 recognition percentage for the $f b$ probe set

\begin{tabular}{|l|lllll|lllll|l|}
\hline \multirow{2}{*}{ Algorithm } & \multicolumn{9}{|c|}{ JPEG coded images - bitrate [bpp] } & \multicolumn{3}{c|}{ JPEG2000 coded images - bitrate [bpp] } & \multicolumn{2}{l|}{ Original } \\
& 0.1 & 0.2 & 0.5 & 0.8 & 1 & 0.1 & 0.2 & 0.5 & 0.8 & 1 & images \\
\hline PCA+L1 & 76.2 & 80.0 & 80.7 & $\mathbf{8 1 . 0}$ & 80.9 & 80.5 & 80.6 & $\mathbf{8 1 . 0}$ & $\mathbf{8 1 . 0}$ & 80.8 & 80.9 \\
PCA+L2 & 78.7 & 80.1 & 81.1 & 81.3 & 81.3 & 81.0 & 81.4 & 81.1 & 81.2 & 81.1 & 81.4 \\
PCA+cos & 76.9 & 80.1 & $\mathbf{8 0 . 5}$ & $\mathbf{8 0 . 5}$ & $\mathbf{8 0 . 5}$ & $\mathbf{8 0 . 5}$ & $\mathbf{8 0 . 5}$ & $\mathbf{8 0 . 6}$ & $\mathbf{8 0 . 6}$ & $\mathbf{8 0 . 5}$ & 80.4 \\
ICA1+L1 & 76.9 & 79.8 & 80.0 & 80.0 & $\mathbf{8 0 . 2}$ & 80.0 & 79.9 & 80.0 & 80.0 & 80.0 & 80.1 \\
ICA1+L2 & 77.5 & 79.9 & $\mathbf{8 0 . 1}$ & $\mathbf{8 0 . 2}$ & $\mathbf{8 0 . 1}$ & 79.9 & 80.0 & 80.0 & $\mathbf{8 0 . 2}$ & $\mathbf{8 0 . 3}$ & 80.1 \\
ICA1+cos & 76.1 & $\mathbf{8 0 . 2}$ & $\mathbf{8 0 . 4}$ & $\mathbf{8 0 . 3}$ & 80.1 & 79.8 & $\mathbf{8 0 . 5}$ & $\mathbf{8 0 . 3}$ & $\mathbf{8 0 . 3}$ & $\mathbf{8 0 . 4}$ & 80.1 \\
ICA2+L1 & 51.2 & 62.0 & 65.1 & 64.8 & 65.0 & 64.0 & 64.6 & 65.0 & $\mathbf{6 5 . 3}$ & 65.0 & 65.3 \\
ICA2+L2 & 58.6 & 70.7 & 73.3 & 73.6 & 73.5 & 71.8 & 73.1 & $\mathbf{7 3 . 8}$ & 73.5 & 73.4 & 73.5 \\
ICA2+cos & 75.2 & 80.7 & $\mathbf{8 2 . 7}$ & 82.3 & $\mathbf{8 2 . 6}$ & 82.1 & $\mathbf{8 2 . 9}$ & $\mathbf{8 2 . 7}$ & $\mathbf{8 2 . 8}$ & $\mathbf{8 2 . 6}$ & 82.3 \\
LDA+L1 & 75.0 & 77.4 & 77.6 & 77.8 & $\mathbf{7 7 . 9}$ & 77.7 & 77.8 & $\mathbf{8 0 . 0}$ & $\mathbf{7 7 . 9}$ & 77.8 & 77.8 \\
LDA+L2 & 79.6 & 81.0 & $\mathbf{8 2 . 4}$ & $\mathbf{8 2 . 5}$ & $\mathbf{8 2 . 4}$ & 82.2 & 82.0 & 82.2 & 82.3 & 82.3 & 82.3 \\
LDA+cos & 77.0 & 80.5 & 81.0 & $\mathbf{8 1 . 1}$ & 81.0 & 81.0 & $\mathbf{8 1 . 3}$ & $\mathbf{8 1 . 4}$ & $\mathbf{8 1 . 2}$ & $\mathbf{8 1 . 2}$ & 81.0 \\
\hline
\end{tabular}

Table 2. Rank 1 recognition percentage for the $f c$ probe set

\begin{tabular}{|l|lllll|llllll|l|}
\hline \multirow{2}{*}{ Algorithm } & \multicolumn{8}{|c|}{ JPEG coded images - bitrate [bp] } & \multicolumn{2}{l}{ JPEG2000 } & coded images - bitrate & \multirow{2}{*}{ Original } \\
& 0.1 & 0.2 & 0.5 & 0.8 & 1 & 0.1 & 0.2 & 0.5 & 0.8 & 1 & \\
& images \\
\hline PCA+L1 & 33.5 & 47.4 & $\mathbf{5 0 . 1}$ & 49.4 & 49.4 & 46.9 & 48.9 & 49.4 & 49.4 & $\mathbf{5 0 . 0}$ & 49.4 \\
PCA+L2 & 21.6 & 24.2 & 23.7 & 24.2 & 24.2 & 23.7 & 24.2 & 24.2 & 24.2 & 24.2 & 24.2 \\
PCA+cos & 13.4 & 18.0 & $\mathbf{1 8 . 5}$ & 18.0 & 18.0 & 18.0 & 18.0 & 18.0 & 18.0 & 18.0 & 18.0 \\
ICA1+L1 & 18.0 & 22.1 & $\mathbf{2 3 . 2}$ & $\mathbf{2 3 . 2}$ & 22.6 & 22.1 & 22.6 & 22.1 & 22.6 & 22.6 & 22.6 \\
ICA1+L2 & 19.0 & 21.6 & 21.6 & $\mathbf{2 2 . 1}$ & 21.6 & 21.6 & 21.6 & 21.6 & $\mathbf{2 2 . 1}$ & $\mathbf{2 2 . 1}$ & 21.6 \\
ICA1+cos & 12.3 & $\mathbf{1 7 . 5}$ & $\mathbf{1 7 . 5}$ & 16.4 & 16.4 & $\mathbf{1 7 . 0}$ & $\mathbf{1 7 . 0}$ & 16.4 & 16.4 & 16.4 & 16.4 \\
ICA2+L1 & 12.3 & 16.4 & 15.9 & 16.4 & 17.5 & 15.9 & 15.9 & 16.4 & 17.5 & 17.0 & 17.5 \\
ICA2+L2 & 22.6 & 39.1 & 40.7 & 41.7 & 41.7 & 39.1 & 40.2 & 41.7 & 41.7 & 41.7 & 41.7 \\
ICA2+cos & 42.2 & 64.4 & 64.4 & 64.4 & 64.4 & 62.8 & $\mathbf{6 4 . 9}$ & 63.9 & 64.4 & 63.9 & 64.4 \\
LDA+L1 & 17.5 & 22.1 & 21.6 & 22.1 & 22.1 & 19.5 & 20.6 & 22.1 & 22.1 & 22.1 & 22.1 \\
LDA+L2 & 22.1 & 25.7 & 26.2 & 26.2 & 26.2 & 25.2 & 25.7 & 26.2 & $\mathbf{2 6 . 8}$ & $\mathbf{2 6 . 8}$ & 26.2 \\
LDA+cos & 14.4 & 19.0 & 19.5 & 19.5 & 19.0 & 18.5 & 19.0 & 19.5 & 19.5 & 19.5 & 19.5 \\
\hline
\end{tabular}

Many cases where performance goes up with compression are observed for the $f b$ probe set (Table 1). For more difficult tasks, the improvement with compression in less often. For the dupl set (Table 3) with images compressed using JPEG2000 at 0.2 bpp there is an improvement in almost all algorithms. The trend continues for dup2 set (Table 4), but is not so emphasized. The fact that performance, almost persistently, goes up with JPEG2000 compression for dupl and dup2 set indicates that JPEG2000 compression is able to efficiently eliminate the differences between original images and the ones taken later in time. Besides, our results show that this effect is consistent 
Table 3. Rank 1 recognition percentage for the $d u p 1$ probe set

\begin{tabular}{|l|lllll|lllll|c|}
\hline \multirow{2}{*}{ Algorithm } & \multicolumn{9}{|c|}{ JPEG coded images - bitrate [bpp] } & \multicolumn{3}{c|}{ JPEG2000 coded images - bitrate [bpp] } & Original \\
& 0.1 & 0.2 & 0.5 & 0.8 & 1 & 0.1 & 0.2 & 0.5 & 0.8 & 1 & images \\
\hline PCA+L1 & 33.5 & 36.0 & 36.9 & 36.9 & 36.9 & 36.7 & $\mathbf{3 7 . 4}$ & $\mathbf{3 7 . 4}$ & 36.9 & 37.1 & 37.1 \\
PCA+L2 & 32.8 & 33.1 & 33.8 & $\mathbf{3 3 . 9}$ & $\mathbf{3 3 . 8}$ & 33.2 & $\mathbf{3 3 . 9}$ & $\mathbf{3 3 . 9}$ & 33.8 & 33.8 & 33.8 \\
PCA+cos & 31.3 & 33.3 & 33.2 & $\mathbf{3 3 . 5}$ & $\mathbf{3 3 . 5}$ & 33.3 & $\mathbf{3 3 . 9}$ & $\mathbf{3 3 . 5}$ & $\mathbf{3 3 . 5}$ & $\mathbf{3 3 . 5}$ & 33.3 \\
ICA1+L1 & 31.5 & $\mathbf{3 2 . 5}$ & $\mathbf{3 2 . 5}$ & 32.4 & 32.4 & 32.5 & $\mathbf{3 2 . 5}$ & 32.4 & 32.2 & 32.2 & 32.4 \\
ICA1+L2 & 31.4 & 32.5 & 32.8 & 32.6 & 32.9 & 32.9 & 32.8 & 32.8 & 32.8 & 32.9 & 32.9 \\
ICA1+cos & 30.8 & $\mathbf{3 3 . 9}$ & 33.8 & 33.6 & 33.8 & 33.9 & $\mathbf{3 4 . 0}$ & 33.8 & 33.6 & 33.6 & 33.8 \\
ICA2+L1 & 19.8 & 26.5 & 29.7 & 29.7 & 29.9 & 28.3 & $\mathbf{3 0 . 1}$ & 29.9 & 29.9 & $\mathbf{3 0 . 0}$ & 29.9 \\
ICA2+L2 & 22.3 & 29.2 & 32.1 & 32.2 & 32.4 & 30.1 & 32.2 & 32.4 & 31.9 & 32.1 & 32.5 \\
ICA2+cos & 34.0 & 39.2 & 43.0 & 42.5 & 42.8 & 40.5 & 42.5 & 42.2 & $\mathbf{4 3 . 0}$ & $\mathbf{4 3 . 0}$ & 42.8 \\
LDA+L1 & 31.8 & 32.8 & $\mathbf{3 3 . 5}$ & $\mathbf{3 3 . 5}$ & $\mathbf{3 3 . 5}$ & 34.3 & $\mathbf{3 3 . 6}$ & $\mathbf{3 3 . 5}$ & $\mathbf{3 3 . 6}$ & 33.3 & 33.3 \\
LDA+L2 & 33.1 & 32.9 & 33.2 & 33.2 & 33.2 & 32.6 & $\mathbf{3 3 . 3}$ & $\mathbf{3 3 . 3}$ & 33.2 & 33.2 & 33.2 \\
LDA+cos & 31.1 & 33.2 & 33.2 & 33.3 & 33.3 & $\mathbf{3 3 . 5}$ & $\mathbf{3 3 . 5}$ & 33.3 & 33.3 & 33.3 & 33.3 \\
\hline
\end{tabular}

Table 4. Rank 1 recognition percentage for the dup2 probe set

\begin{tabular}{|l|ccccc|ccccc|c|}
\hline \multirow{2}{*}{ Algorithm } & \multicolumn{9}{|c|}{ JPEG coded images - bitrate [bpp] } & \multicolumn{3}{c|}{ JPEG2000 coded images - bitrate [bpp] } & Original \\
& 0.1 & 0.2 & 0.5 & 0.8 & 1 & 0.1 & 0.2 & 0.5 & 0.8 & 1 & images \\
\hline PCA+L1 & 14.1 & 17.5 & 17.9 & 17.9 & 17.5 & 17.1 & $\mathbf{1 8 . 3}$ & $\mathbf{1 8 . 3}$ & 17.5 & 17.9 & 17.9 \\
PCA+L2 & 9.8 & 9.8 & 10.2 & 10.2 & 10.2 & 8.5 & 10.2 & 10.2 & 10.2 & 10.2 & 10.2 \\
PCA+cos & 10.2 & 11.1 & 10.6 & 11.1 & 11.1 & 10.6 & 11.1 & 11.1 & 11.1 & 11.1 & 11.1 \\
ICA1+L1 & 10.6 & $\mathbf{1 1 . 9}$ & 11.1 & 11.1 & 11.1 & 10.6 & 11.1 & 11.1 & 11.1 & 11.1 & 11.1 \\
ICA1+L2 & 10.2 & 10.6 & 11.1 & 10.6 & 11.1 & 10.2 & 10.6 & 10.6 & 11.1 & 11.1 & 11.1 \\
ICA1+cos & 10.2 & 11.9 & 12.3 & 12.3 & 12.3 & 11.5 & 11.9 & 12.3 & 12.3 & 12.3 & 12.3 \\
ICA2+L1 & 12.3 & 14.5 & $\mathbf{1 6 . 6}$ & 16.2 & 16.2 & 16.2 & $\mathbf{1 8 . 3}$ & $\mathbf{1 6 . 6}$ & $\mathbf{1 6 . 6}$ & $\mathbf{1 6 . 6}$ & 16.2 \\
ICA2+L2 & 14.1 & 17.5 & 18.8 & 19.2 & 18.8 & 17.9 & $\mathbf{1 9 . 6}$ & 18.8 & 18.8 & 18.8 & 19.2 \\
ICA2+cos & 21.3 & 25.2 & 26.9 & 26.9 & 27.7 & 23.5 & 26.9 & 26.5 & $\mathbf{2 7 . 7}$ & 26.9 & 27.3 \\
LDA+L1 & 12.3 & $\mathbf{1 4 . 1}$ & $\mathbf{1 4 . 5}$ & $\mathbf{1 3 . 6}$ & 13.2 & $\mathbf{1 4 . 1}$ & $\mathbf{1 4 . 5}$ & $\mathbf{1 4 . 5}$ & 13.2 & 13.2 & 13.2 \\
LDA+L2 & $\mathbf{1 0 . 2}$ & $\mathbf{9 . 8}$ & 9.4 & 9.4 & 9.4 & 8.9 & $\mathbf{1 0 . 2}$ & $\mathbf{9 . 8}$ & 9.4 & 9.4 & 9.4 \\
LDA+cos & 9.4 & 10.6 & 10.2 & $\mathbf{1 0 . 6}$ & 10.2 & $\mathbf{1 0 . 6}$ & 10.2 & $\mathbf{1 0 . 6}$ & $\mathbf{1 0 . 6}$ & $\mathbf{1 0 . 6}$ & 10.2 \\
\hline
\end{tabular}

across almost all algorithms, and it indicates that the information eliminated is not algorithm specific but is a property of compression of those images. The $f c$ test (Table 2) turns out to be the most difficult one regarding performance improvement with compressed images. Obviously, both JPEG and JPEG2000 compression techniques eliminate the important information for illumination changes less efficiently than for changes induced by images taken later in time. We can make an ad hoc assumption as to why is this so by looking at the images in Figure 1. Obviously, when the original image is compressed, the minor differences caused by different expression and/or temporal changes are reduced. For example, the images compressed using 
JPEG2000 look a bit "smeared". The fact that both JPEG and JPEG2000 are low-pass filters in some sense could explain the improvements in $f b$, dupl and dup2 tests. Situation with illumination changes is a bit different because the differences in images that arise from different illuminations are larger and affect the whole image. Thus, the low-pass filtering in most cases does not improve performance. In overall, JPEG2000 seems superior in all tests and should be considered as a standard for storing and transmission of face images for biometric purposes.

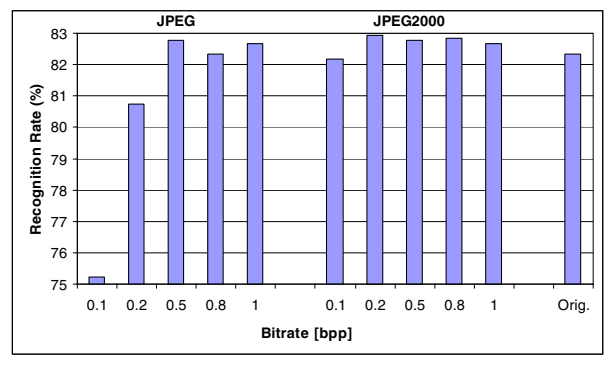

(a)

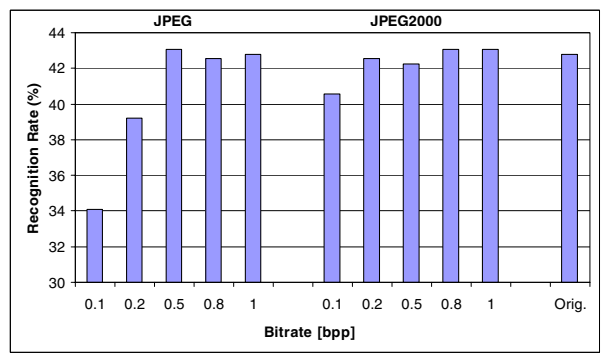

(c)

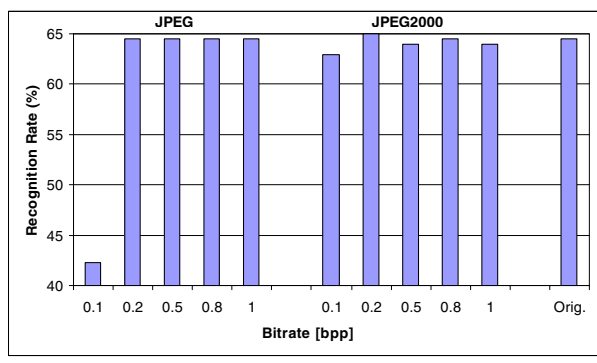

(b)

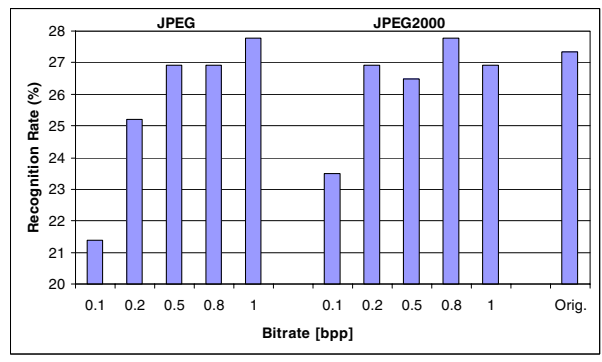

(d)

Fig. 2. ICA2+COS performance as a function of bpp: (a) $f b$ probe set, (b) $f c$ probe set, (c) $d u p l$ probe set, (d) dup2 probe set.

Illustrated by the ICA2+COS algorithm (the best algorithm for all tasks in our experiments) in Figure 2, a significant superiority of JPEG2000 over JPEG on high compression ratios can be seen. For example, if we look at Figure 2(b), we can see that performance for compression ratio of 80:1 (0.1 bpp) using JPEG2000 is only slightly (statistically insignificant difference) lower than with original images. Using JPEG compression for that same case deteriorates performance for more than $20 \%$. Again, this trend is persistent throughout all cases and is therefore a rule in our experiments. All the conclusions brought in the previous text are also clearly illustrated by Figure 2(a)-2(d).

And finally, a word about metrics. Our results show that cosine (angle) metric takes the most advantage out of compression across all algorithms (you can easily confirm this by looking at the bolded values in Table 1). The reason for this behaviour stays unclear. 


\section{Conclusions and Further Work}

In this paper we explored the effects of JPEG and JPEG2000 compression techniques on face recognition algorithm performance. This is the first comprehensive study of standard JPEG2000 compression effects on face recognition, as well as an extension of existing experiments for JPEG compression. A wide range of bitrates (compression ratios) was used on probe images and results are reported for 12 different subspace face recognition algorithms. We found that not only that compression does not deteriorate performance but it, in some cases, even improves it slightly. We believe that this is more than enough reason to further explore the theoretical effects of compression on face recognition and eventually to use some compression scheme for storing and transmission of face images used as a biometric (JPEG2000 seems like a reasonable choice by our experiments).

The future perspectives of using standard surveillance equipment as input into superior performance recognition systems are becoming a reality. Storing compressed face images on low-capacity chips, ID and smart cards are a reality also. A general belief that the effect of compression in machine vision applications is deleterious is proven to be questionable by this study.

Our further research will focus on explaining the noticed unexpected effects, like the effect that JPEG2000 is able to efficiently capture information essential for recognizing changes caused by differences between images taken later in time or that cosine metric seems to take the most advantage of compression. Quantifying image quality (for compressed and uncompressed images) prior to using it as input to recognition systems, in a way that the best image be chosen as input, is another subject worth researching.

\section{Acknowledgment}

Portions of the research in this paper use the Color FERET database of facial images collected under the FERET program.

\section{References}

1. W. Zhao, R. Chellappa, J. Phillips, and A. Rosenfeld, "Face Recognition in Still and Video Images: A Literature Survey", ACM Computing Surveys, Vol. 35, Dec. 2003, pp. 399-458

2. M. Turk, A. Pentland, "Eigenfaces for Recognition", Journal of Cognitive Neuroscience, Vol. 3, No. 1, 1991, pp. 71-86

3. M.S. Bartlett, J.R. Movellan, T.J. Sejnowski, "Face Recognition by Independent Component Analysis", IEEE Trans. on Neural Networks, Vol. 13, No. 6, November 2002, pp. 1450-1464

4. P. Belhumeur, J. Hespanha, D. Kriegman, "Eigenfaces vs. Fisherfaces: Recognition Using Class Specific Linear Projection", Proc. of the Fourth European Conference on Computer Vision, ECCV'96, Vol. 1, 15-18 April 1996, Cambridge, UK, pp. 45-58

5. G. Wallace, "The JPEG Still-picture Compression Standard", Communications of the ACM Vol. 34, No. 4, 1991, pp. 31-44 
6. C. Chistopoulos, A. Skodras, T. Ebrahimi, "The JPEG2000 Still Image Coding System: An Overview", IEEE Trans. on Consumer Electronics, Vol. 46, November 2000, pp. 11031127

7. D.M. Blackburn, J.M. Bone, P.J. Phillips, "FRVT 2000 Evaluation Report", February 2001, Available: http://www.frvt.org/FRVT2000/documents.htm

8. K. Wat, S.H. Srinivasan, "Effect of Compression on Face Recognition", Proc. of the 5th International Workshop on Image Analysis for Multimedia Interactive Services, WIAMIS 2004, 21-23 April 2004, Lisboa, Portugal

9. H. Moon, P.J. Phillips, "Computational and Performance Aspects of PCA-based Facerecognition Algorithms", Perception, Vol. 30, 2001, pp. 303-321

10. P.J. Phillips, H. Moon, S.A. Rizvi, P.J. Rauss, The FERET Evaluation Methodology for Face Recognition Algorithms, IEEE Trans. on Pattern Analysis and Machine Intelligence, Vol. 22, No. 10, October 2000, pp. 1090-1104

11. Independent JPEG Group's JPEG software packet, Available: ftp:/ftp.uu.net/graphics/ jpeg/jpegsrc.v6b.tar.gz

12. Kakadu JPEG2000 software, Available:http://www.kakadusoftware.com/win32_ executables.zip

\section{Appendix}

Hypothesis testing using McNemar's test on results of comparisons in Tables 1 - 4. Here we report $p$-values for performance of a given algorithm on original images and the best case for compressed images (p-1) and between performance on original images and the worst case for compressed images ( $\mathrm{p}-2)$. Table 5 gives results for JPEG compression and Table 6 for JPEG2000. When $p$-value is higher than 0.05 (standard cut-off) the difference in performance is statistically insignificant.

Table 5. $p$-values obtained by McNemar's test for results with JPEG compression

\begin{tabular}{|c|cc|cc|cc|cc|}
\hline \multirow{2}{*}{ Algorithm } & \multicolumn{2}{|c|}{$f b$ probe set } & \multicolumn{2}{c|}{$f c$ probe set } & \multicolumn{2}{c|}{ dup1 probe set } & \multicolumn{2}{c|}{$d u p 2$ probe set } \\
\cline { 2 - 10 } & $\mathrm{p}-1$ & $\mathrm{p}-2$ & $\mathrm{p}-1$ & $\mathrm{p}-2$ & $\mathrm{p}-1$ & $\mathrm{p}-2$ & $\mathrm{p}-1$ & $\mathrm{p}-2$ \\
\hline PCA+L1 & 0.50 & $10^{-12}$ & 0.50 & $10^{-10}$ & 0.50 & $10^{-4}$ & 1.00 & 0.03 \\
PCA+L2 & 0.50 & $10^{-5}$ & 0.75 & 0.15 & 0.50 & 0.13 & 1.00 & 0.50 \\
PCA+cos & 0.25 & $10^{-7}$ & 0.50 & 0.02 & 0.50 & $10^{-3}$ & 0.50 & 0.31 \\
ICA1+L1 & 0.25 & $10^{-7}$ & 0.50 & 0.01 & 0.50 & 0.19 & 0.25 & 0.50 \\
ICA1+L2 & 0.31 & $10^{-5}$ & 0.50 & 0.13 & 1.00 & 0.03 & 1.00 & 0.36 \\
ICA1+cos & 0.12 & $10^{-10}$ & 0.25 & 0.01 & 0.50 & $10^{-4}$ & 1.00 & 0.03 \\
ICA2+L1 & 0.37 & $10^{-39}$ & 1.00 & 0.02 & 0.68 & $10^{-17}$ & 0.50 & $10^{-3}$ \\
ICA2+L2 & 0.50 & $10^{-44}$ & 1.00 & $10^{-11}$ & 0.50 & $10^{-18}$ & 1.00 & $10^{-4}$ \\
ICA2+cos & 0.08 & $10^{-15}$ & 1.00 & $10^{-12}$ & 0.36 & $10^{-12}$ & 0.50 & $10^{-3}$ \\
LDA+L1 & 0.50 & $10^{-5}$ & 0.75 & 0.03 & 0.50 & 0.02 & 0.12 & 0.36 \\
LDA+L2 & 0.12 & $10^{-5}$ & 0.50 & 0.03 & 1.00 & 0.50 & 0.50 & 0.34 \\
LDA+cos & 0.50 & $10^{-10}$ & 1.00 & $10^{-3}$ & 1.00 & $10^{-3}$ & 0.50 & 0.34 \\
\hline
\end{tabular}


Table 6. $p$-values obtained by McNemar's test for results with JPEG2000 compression

\begin{tabular}{|c|cc|cc|cc|cc|}
\hline \multirow{2}{*}{ Algorithm } & \multicolumn{2}{|c|}{$f b$ probe set } & \multicolumn{2}{|c|}{$f c$ probe set } & \multicolumn{2}{c|}{ dup1 probe set } & \multicolumn{2}{c|}{ dup2 probe set } \\
\cline { 2 - 9 } & $\mathrm{p}-1$ & $\mathrm{p}-2$ & $\mathrm{p}-1$ & $\mathrm{p}-2$ & $\mathrm{p}-1$ & $\mathrm{p}-2$ & $\mathrm{p}-1$ & $\mathrm{p}-2$ \\
\hline PCA+L1 & 0.50 & 0.21 & 0.50 & 1.00 & 0.36 & 0.31 & 0.50 & 0.31 \\
PCA+L2 & 0.62 & 0.25 & 1.00 & 1.00 & 0.50 & 0.19 & 1.00 & 0.06 \\
PCA+cos & 0.12 & 0.50 & 1.00 & 1.00 & 0.06 & 0.62 & 1.00 & 0.50 \\
ICA1+L1 & 0.68 & 0.38 & 1.00 & 0.50 & 0.50 & 0.50 & 1.00 & 0.50 \\
ICA1+L2 & 0.25 & 0.40 & 0.50 & 1.00 & 0.60 & 0.50 & 1.00 & 0.25 \\
ICA1+cos & 0.12 & 0.26 & 0.50 & 1.00 & 0.31 & 0.50 & 1.00 & 0.25 \\
ICA2+L1 & 0.65 & 0.02 & 0.75 & 0.22 & 0.40 & 0.02 & 0.03 & 0.65 \\
ICA2+L2 & 0.22 & $10^{-3}$ & 1.00 & 0.06 & 0.50 & $10^{-3}$ & 0.50 & 0.18 \\
ICA2+cos & 0.11 & 0.44 & 0.20 & 0.22 & 0.31 & $10^{-3}$ & 0.50 & 0.01 \\
LDA+L1 & 0.31 & 0.50 & 1.00 & 0.03 & 0.25 & 0.07 & 0.18 & 1.00 \\
LDA+L2 & 1.00 & 0.50 & 0.50 & 0.25 & 0.19 & 0.19 & 0.25 & 0.50 \\
LDA+cos & 0.06 & 0.61 & 1.00 & 0.25 & 0.50 & 0.50 & 0.50 & 1.00 \\
\hline
\end{tabular}

